\title{
A Different Approach to Traffic Accidents as the Main Cause of Trauma in Developing Countries
}

\author{
Pezhman Bagheri ${ }^{1,} ;$ Mahdi Moshki $^{2}$ \\ ${ }^{1}$ Department of Education and Research, Jiroft University of Medical Science, Jiroft, IR Iran \\ ${ }^{2}$ Department of Public Health, School of Health; Social Development \& Health Promotion Research Center, Gonabad University of Medical Science, Gonabad, IR Iran \\ ${ }^{*}$ Corresponding author: Pezhman Bagheri, Department of Education and Research, Jiroft University of Medical Sciences, Pasdaran Boulevard, Jiroft, IR Iran, Tel/Fax: + 98-3482318084, \\ E-mail: bpegman@yahoo.com.
}

Received: November 7, 2012; Revised: January 16, 2013; Accepted: February 24, 2013

Keywords: Accidents; Trauma

\section{Dear Editor:}

Traffic accidents cause a high Years of Potential Life Lost (YPLL) and permanent disability, trauma and also considered as a public health topic with a total of 105 million fatalities annually $(1,2)$. Traffic accidents in Iran have always caused most of the deaths. According to the repots, every 26 minutes a person loses his life due to the traffic accidents in Iran. Despite widespread public education through various media and safety plans, traffic accidents take the lives of thousands of Individual $(3,4)$.

I think that traffic accidents can be divided into two categories of hardware and software factors. Based on this, hardware factors include all factors except those related to human resources. Cars and roads characteristics and traffic tools are the most important hardware factors. Based on the above definition, software factors are those related to human beings and their conception of driving. Not to obey the law, underlying mental illness, neglect of law enforcement, lack of financial infrastructure, lack of proper preventive rules and wrong policies of the country macro planners are the most visible examples of software factors.

It seems that considering scientific, technological, and hardware advances in the current situation, the focal point of attention and preventive measures are software factors. It was the senior policy makers' opinion for a long time that car accidents and crimes could be decreased by providing physical infrastructure and eliminating physical obstacles. However, after the physical transformation in the form of "hardware transition", software factors are the turning point of overcoming nowadays uncontrollable cars accidents.

In less developed countries most of attention is paid to speed more than other factors, but in more developed countries observing rules and drivers' behavior toward other drivers and pedestrians as an important software strategy, is considered important and educational needs on this issue are provided. Legislation and implementation of accurate and without consideration of pedestrians' laws is very effective in reducing accidents within the city, which is ignored at present. It seems that software factors are the main ones in the current situation. One of the important software factors which increases the risk undertake of people and reduces their risk aversion is inappropriate rules related to car insurance policies. Many existing laws of cars insurance reduce the risk aversion of drivers, which should be overviewed based on drivers' individual factors and his or her records. In addition, given the present situation, law weaknesses and lack of individual deterrence play roles as two basic causes of traffic accidents in less developed countries, and the current rate of traffic offenses is not disincentive and cannot guarantee the promotion of driving culture in these countries. Proper and targeted training of driving, providing appropriate legal action to hold periodical training courses for all drivers, and screening drivers breaking the law regulations from those respecting, imposing financial penalties commensurate with economic and humanistic losses of traffic violations, revision of existing expired legislations, providing preventive judicial legislations, training caring professional executives and trying to identify and cure the offending drivers' psychological problems are the most important software strategies. In addition, providing standard physical infrastructure in the traffic paths, developing and applying safety principles and high-risk aversion of vehicles, traffic flow restrictions of vehicles categorized for different and separate routes based on their kind, absolute prohibition of traffic for accident making drivers are the most 
important hardware strategies. It is hoped that development of scientific exchanges between different cultures and countries and everyone's feel of need to change the driving culture and adoption of rules to suit local conditions in each region with the approach to obstacles and software strategies can manage the crisis of uncontrollable traffic accidents around the world, especially in less developed countries.

\section{Acknowledgements}

There is no Acknowledgment.

\section{Authors' contribution}

All the authors contributed in the writing and research work.

\section{Financial disclosure}

The authors declared no financial interests related to the manuscript materials.

\section{References}

1. Peden R, Scurfield D, Sleet D, Mohan A, Hyder A, Jarawan E. World report on road traffic injury prevention.: Geneva: World Health Organization; 2004.

2. Naghavi M. Death features in 23 Provinces of Iran.Tehran: Ministry of Health and Medical Education; 2005.

3. Ali M, Saeed MM, Ali MM, Haidar N. Determinants of helmet use behaviour among employed motorcycle riders in Yazd, Iran based on theory of planned behaviour. Injury. 2011;42(9):864-9.

4. Kadkhodaie MH. Three-year review of facial fractures at a teaching hospital in northern Iran. Br J Oral Maxillofac Surg. 2006;44(3):229-31. 